\title{
Análisis de cambio de cobertura y uso de suelo en una subcuenca preandina chilena. Herramienta para la sustentabilidad productiva de un territorio'
}

\author{
Alejandra Sepúlveda-Varas², Pablo Saavedra-Briones ${ }^{3}$ y Carlos Esse ${ }^{4}$
}

\begin{abstract}
RESUMEN
Se analizó la dinámica de cambio de cobertura y uso de suelo para una subcuenca hidrográfica, región de La Araucanía, Chile, periodo 1994-2007. El área registra cambios en la cobertura del suelo, intensa división predial y degradación de los sistemas naturales. La metodología comprendió (1) análisis cartográfico, matriz de confusión e índice de kappa y (2) análisis/cuantificación de cambios mediante matriz de cambio. Los resultados permitieron identificar áreas sometidas a mayor presión de uso, identificándose las categorías "cultivos-praderas naturales" y "plantaciones forestales" como las coberturas que concentran el 79,5\% de la superficie total del cambio neto en el área, con tasa de ganancia de superficie de uso forestal con especies exóticas de 432 ha año-1 en desmedro de suelos de uso agropecuario. Nuestros resultados reafirman la necesidad de analizar la evolución en el uso del suelo previo a la identificación de las potencialidades del recurso suelo para soportar nuevas actividades productivas.
\end{abstract}

Palabras claves: Sustentabilidad, Uso de la tierra y Cobertura, Uso de suelo.

\begin{abstract}
The dynamics of change in soil use, period 1994-2007, for a watershed in the region of La Araucania, Chile, was analyzed. The study area shows changes in soil use, intense land division and degradation of natural systems. The methodology included (1) cartographic analysis, confusion matrix and kappa index, and (2) analysis/quantification of changes using a change matrix. The results allowed us to identify spatially the soil covers subject to the greatest use pressure. "Crops and natural grassland" and "forestry plantations" were the categories identified as accounting for $79,5 \%$ of the total area undergoing soil use change in the study area, with a surface profit rate of forest use exotic species of 432 ha year ${ }^{-1}$ at the expense of soil for agricultural use. Our results reaffirm the need to analyze the evolution in soil use prior to the identification of the potential of soil resources to support new productive activities.
\end{abstract}

Keywords: Sustainability, Land use and land cover, Soil use. 
Alrededor de la mitad de la superficie terrestre ha sido directamente transformada por acción humana en función de cubrir las necesidades del hombre (Hooke et al., 2012). A escala global, se estima que entre los años 1700 y 1992, 1.621 millones de hectáreas fueron habilitadas para la agricultura, de ellas 885 millones correspondían a bosques, 565 millones a sabana, praderas y estepa, 150 millones a matorrales y 21 millones a tundras y desiertos (Ramankutty \& Foley 1999). En la mayoría de los países, el principal cambio de cobertura de suelo, en una perspectiva extendida en el tiempo, es la conversión de superficie forestal a cobertura y uso agrícola (Barbier et al., 2010). En este sentido, se reconoce a la actividad humana como la mayor responsable de los cambios contemporáneos en los flujos y estados de la biósfera (Nájera et al., 2010; Mohammad \& Mohammad, 2010; Ouyang et al., 2010; Marín et al., 2011; Carmona \& Nahuelhual, 2012).

El conocimiento de la cobertura y uso del suelo constituye uno de los aspectos más importantes dentro del análisis físico biótico para el ordenamiento del territorio (Aguayo et al., 2009), tanto por su influencia en la formación y evolución de los suelos, y el impacto del cambio de cobertura de suelo sobre la provisión de servicios ecosistémicos (Farley, 2007). A escala local, estos cambios inducen el deterioro y degradación de suelos, así como modificaciones en nichos ecológicos y resiliencia de los componentes ambientales (Nájera et al., 2010). Oehl et al. (2010) demuestran que la intensidad de uso del suelo por sobre el tipo de suelo y composición vegetacional condiciona la composición y permanencia de comunidades de hongos micorrízicos impactando con ello la calidad biológica del suelo. A escala regional, la modificación en cobertura y uso del suelo, altera los ciclos hídricos de las cuencas, los regímenes de temperatura, el secuestro de dióxido de carbono y la biodiversidad (Nájera et al., 2010), así como el aumento en la vulnerabilidad de grupos humanos. Globalmente, el cambio de uso del suelo se ha identificado como uno de los principales agentes forzantes del cambio climático (Foley et al., 2005).

Para una adecuada caracterización de la cobertura y uso de suelo en un territorio, la elección de los límites de la zona a cartografiar depende del objetivo del estudio (Reyes et al., 2006). Debido a que el ciclo hidrológico es la matriz en la que funcionan todos los otros ciclos biogeoquímicos (Chapin et al., 2005), las cuencas hidrográficas son un marco espacial adecuado para el estudio de los ecosistemas. Estas constituyen una unidad funcional e integral con límites precisos y salidas puntuales (Maass \& Cotler, 2008) que permiten el manejo integrado de los bienes y servicios que proveen los ecosistemas, los cuáles resultan alterados al modificarse el uso y cobertura del suelo.

Respecto al análisis del cambio de uso de suelo con énfasis en la cobertura forestal, varios autores utilizan la teoría de transición forestal (Bae et al., 2012; Barbier et al., 2010; Mather, 2007; Rudel et al., 2005), requiriéndose una distinción en su aplicación para países desarrollados respecto a países en vías de desarrollo (Farley, 2007). Para el segundo grupo, la causa del aumento en la cobertura forestal se explica por la llamada "vía de escases forestal" en la que, dado el mayor precio de los productos forestales, los agricultores o dueños de la tierra abandonan la actividad agropecuaria reconvirtiéndose a la actividad forestal (Barbier et al., 2010; Farley, 2007; Rudel et al., 2005), expandiéndose la cobertura forestal mediante plantación de árboles en áreas desprovistas de ellos (aforestación). Así, el concepto de transición de uso del suelo se refiere 
a cualquier cambio ocurrido en el uso del suelo transitando desde un estado a otro (Lambin \& Meyfroidt, 2010).

En Chile, la implementación del "Catastro y Evaluación de los Recursos Vegetacionales Nativos de Chile" por parte de CONAF-CONAMA-BIRF (1999) permite cuantificar los recursos vegetacionales nativos del país y ejecutar una serie de monitoreos nacionales y regionales para detectar los cambios de cobertura de suelo. En la región de La Araucanía, Chile, el primer monitoreo y actualización de cambios comprende el período 1993-2007, ilustrándose la fuerte presión de uso de suelo debido a cambios en el patrón productivo (CONAF-CONAMA, 2009).

Sin duda, se hace relevante contar con información actualizada que permita identificar los cambios en la cobertura del suelo ocurridos en un territorio -posibles de ser determinados mediante una metodología robusta- $y$, mediante ello, analizar la relación entre actividades productivas y uso del suelo para contribuir a la toma de decisiones para un uso sustentable de los recursos naturales en el territorio. Esta relación se vincula con los mecanismos causales asociados al cambio de uso de suelo, pudiendo distinguirse dos tipos: socioecológico (modificación del uso de suelo como resultado de la pérdida de servicios ecosistémicos) y socioeconómico (modificación del uso del suelo como resultado de cambios en el entorno productivo externo) (Lambin \& Meyfroidt, 2010).

En este sentido, nuestra hipótesis de trabajo postula que cambios en los agroecosistemas son posibles de constatar mediante la identificación de los patrones de uso de suelo y con ello identificar la dinámica de uso. De esta manera, el objetivo de la presente investigación es determinar la evolución temporal de la cobertura del suelo para la subcuenca del río Quepe, en el periodo 1994-2007, mediante la caracterización de variables de uso y cobertura de suelo.

\section{Metodología propuesta para el análisis de la subcuenca preandina del río Quepe}

El área de estudio se ubica en la región de La Araucanía, entre los $38^{\circ} 40^{\prime}$ y $39^{\circ} 00^{\prime}$ de Latitud Sur y entre los $71^{\circ} 45^{\prime}$ y $73^{\circ}$ 00' de Longitud Oeste, abarca una superficie de $1.604 \mathrm{~km}^{2}$ y se extiende por 110 kilómetros en la depresión intermedia, atravesando la comunas de Vilcún, Padre Las Casas, Freire y Nueva Imperial donde se une al río Cautín. Domina el clima templado cálido Iluvioso con influencia mediterránea. La temperatura media anual es de $12^{\circ} \mathrm{C}$ con una humedad relativa de un $80 \%$ y una precipitación media anual de $1.325 \mathrm{~mm}$ (Di Castri \& Hajek, 1976; PLADECO, 2010a; PLADECO, 2010b; CADE-IDEPE, 2004). Los suelos se agrupan mayoritariamente en el orden Andisol derivados de ceniza volcánica, profundos, con alta retención de agua (40\% a 60\% a $33 \mathrm{kpa})$, elevada porosidad total (60\% a 80\%) y buen drenaje interno (CADE-IDEPE, 2004).

Se estableció una zonificación en base a la división político administrativa de la subcuenca, respecto a la cual se distinguieron: (1) Zona Alta (ZA), correspondiente a la comuna de Vilcún; (2) Zona Media 1 (ZM1), correspondiente a la comuna de Padre Las Casas; (3) Zona Media 2 (ZM2), correspondiente a la comuna de Freire y (4) Zona Baja (ZB), correspondiente a la comuna de Nueva imperial (Figura $\mathrm{N}^{\circ} 1$ ). 
Figura $N^{\circ} 1$

Área de estudio, subcuenca del río Quepe, región de La Araucanía, Chile.
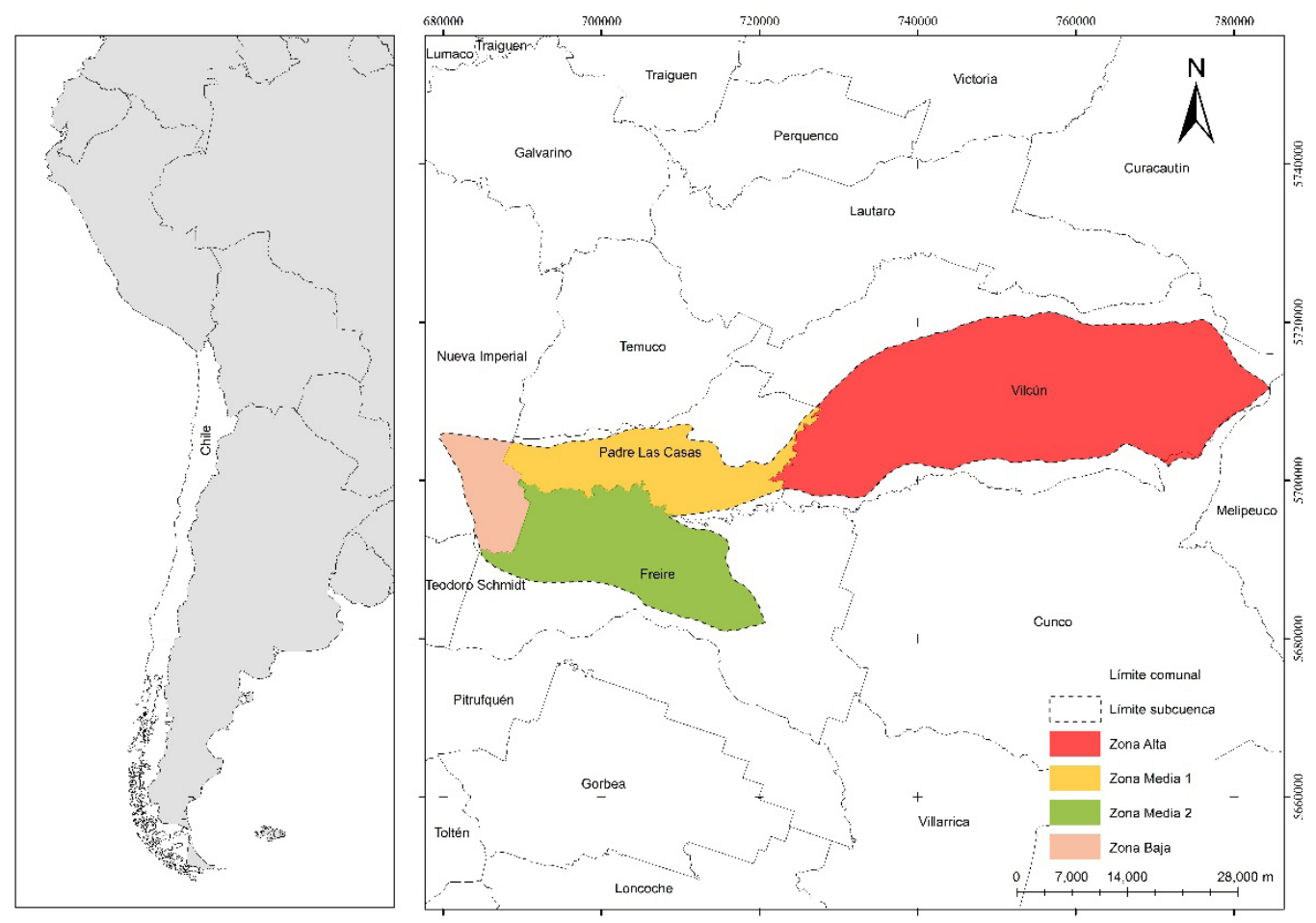

Fuente: Elaboración propia.

\section{Variables de uso}

Se recopiló información referente a las características del sistema humano de la subcuenca, identificando los principales rubros y sistemas productivos existentes en ella. La búsqueda incluyó información (1) inédita como publicaciones y artículos de revistas científicas, e (2) inédita como bases de datos, tesis de grado y documentos e informes de organismos estatales, privados y organizaciones no gubernamentales.

\section{Análisis cartográfico}

La delimitación cartográfica de la subcuenca se basó en DGA (2004). Las coberturas de suelo fueron obtenidas de las bases cartográficas de (1) CONAF-CONAMA-BIRF (1999) y CONAF-CONAMA (2009), (2) Gobierno Regional de La Araucanía versión 2011, (3) Distrito del Manejo del Fuego de CONAF versión 2010 y (4) IGM versión 2007. Las coberturas fueron revisadas y homologadas a proyección UTM, datum WGS 84, huso 18 Sur extendido y procesadas en el software ARCGIS 10.2 (ESRI, EE.UU). En tanto, las clases de cobertura se construyeron en base a una simplificación y agregación del "Sistema General de Clasificación de la Vegetación" desarrollado por CONAF-CONAMA-BIRF (1999). Los productos cartográficos se validaron mediante la elaboración de una matriz de confusión a partir de 100 puntos de control distribuidos al azar en la subcuenca y obtención de índice de kappa (Chuvieco, 2008). 


\section{Análisis y cuantificación de cambios}

La trayectoria y la magnitud del cambio de uso de suelo se determinaron utilizando una matriz de cambio que consiste en una tabla simétrica de doble entrada, ubicándose en cada celda la superficie de coberturas que experimentaron cambios entre el período 1994 y 2007 (Aguayo et al. 2009). La construcción y análisis de la matriz de cambio, siguió la metodología propuesta por Pontius et al. (2004) y sistematizada por Braimoh (2006), que basa el análisis de acuerdo a variación neta, ganancias brutas y pérdidas brutas. Dichos análisis se realizaron a partir de la base cartográfica del Catastro y Evaluación de los Recursos Vegetaciones Nativos de Chile y Catastro de Uso del Suelo y Vegetación: Monitoreo y actualización para la región de La Araucanía, unidad mínima cartografiable de 6,25 ha (CONAF-CONAMA-BIRF, 1999; CONAF-CONAMA, 2009).

\section{Evolución temporal de la cobertura de suelo}

\section{Variables de uso}

La subcuenca del río Quepe presenta tres rubros dominantes: agrícola, forestal y ganadero. Los balances de producción y superficie de los principales cultivos de la subcuenca se muestran en la Figura $\mathrm{N}^{\circ} 2$. En general, los balances de producción (Figura $\mathrm{N}^{\circ} 2 \mathrm{2}$ ) fueron positivos, aunque la superficie agrícola del área de estudio disminuyó su magnitud en 9.185 ha entre el VI y VII Censo agropecuario y forestal (Figura $\mathrm{N}^{\circ} 2 \mathrm{~b}$ ). La independencia de los valores de superficie de cultivos y su producción anual puede explicarse debido al incremento de los rendimientos por hectárea para cada tipo cultivo, los cuales aumentaron notablemente, llegando incluso a duplicarse durante el periodo intercensal 1997-2007 (INE, 2007; INE-ODEPA, 2007; INE, 2008; ICET-ODEPA).

Figura $\mathrm{N}^{\circ} 2$

Balance de producción en quintales métricos (Qmt) (2a) y de superficie en hectáreas (ha) (2b) de los principales cereales cultivados en la subcuenca del río Quepe, región de La Araucanía, para el periodo

1997-2007.
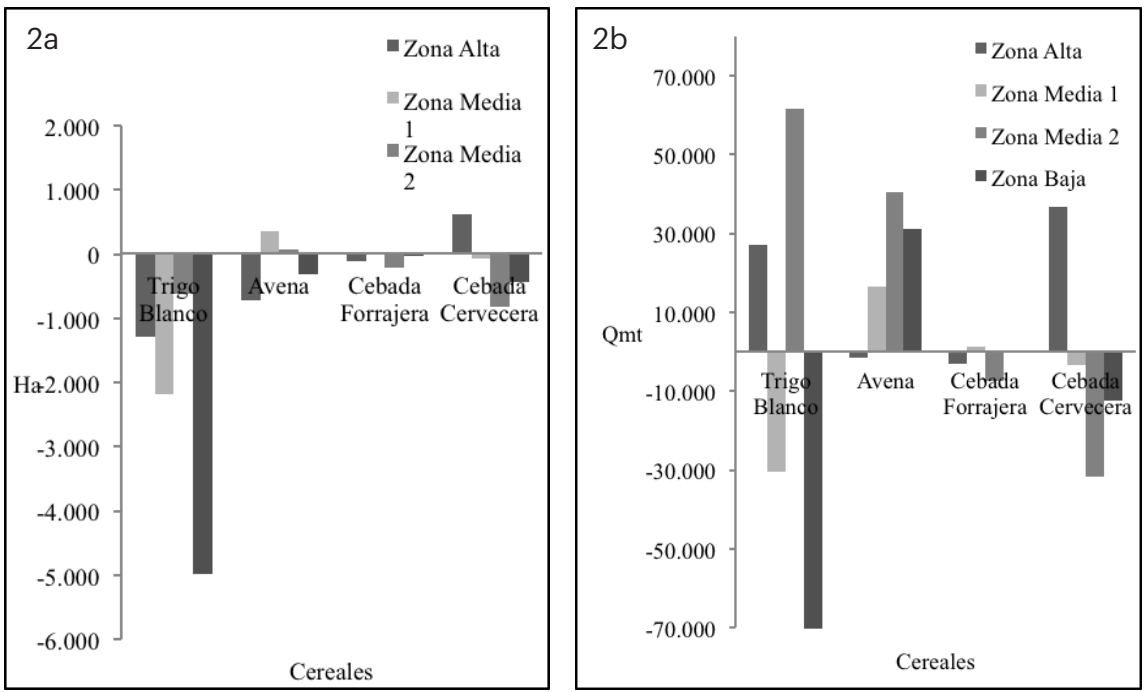

Fuente: Elaboración propia. 
El sector agrícola se caracteriza por el cultivo -en su mayoría para la agroindustria- de avena, maíz, cebada cervecera y forrajera, centeno, trigo blanco y candeal y triticale; donde el trigo blanco y la avena presentan las mayores superficies cultivadas a nivel intercomunal entre el periodo intercensal 1997-2007. La actividad ganadera incluye animales destinados a la reproducción, trabajo y alimentación. Es así como la crianza considera a bovinos, ovinos, porcinos, caprinos, equinos y camélidos (ganado mayor), también aves de criaderos y caseras (ganado menor) y leche, lana y huevos (productos pecuarios). La categoría de ganado más abundante es el bovino (60\%) seguido por el ganado ovino (21\%). La actividad forestal se desarrolla a través de plantaciones forestales, manejo de bosque nativo, obtención de madera pulpable, aserrable y productos forestales no maderables; Pinus radiata D. Don es la especie más abundante con $56 \%$ de la superficie forestal exótica.

El trigo blanco constituye el cultivo anual de mayor extensión en el área de estudio (INE, 2008). En la ZA para el periodo 1997-2007, la producción de trigo blanco aumentó 27.055 qmt (quintales métricos), el rendimiento lo hizo en $12,4 \mathrm{gmt} /$ ha y la superficie de cultivo experimentó una reducción de 1.292,6. En la ZM2, la producción de trigo aumentó a 360.102 qmt en 2007, lo que significa un incremento de 61.738 qmt entre el periodo intercensal, el rendimiento por hectárea subió a $57,3 \mathrm{qmt} / \mathrm{ha}$ y la superficie de cultivo de redujo en 616,6 ha respecto a 1997. La ZM1, en tanto, redujo su producción en 30.330 qmt entre 1997 y 2007. El rendimiento del trigo blanco en la ZM1 aumentó de 21,2 qmt/ha en 1997 a 25,85 qmt/ha en 2007, mientras que la superficie de cultivo tuvo una contracción de $2.177,7$ ha. La ZB, por su parte, experimentó una caída en la producción y superficie cultivada de trigo blanco de 78.892 qmt y 4.973,2 ha respectivamente; sin embargo, el rendimiento aumentó $10 \mathrm{gmt} / \mathrm{ha}$.

El segundo cultivo de mayor superficie lo constituye la avena de grano seco (INE, 2008). Para el período de 1997-2007, la ZA experimentó una reducción de 1.536 qmt en la producción del cereal y de 713,6 ha de superficie cultivada; el rendimiento en tanto aumentó en 6,9 qmt/ha. Para la ZM2, tanto producción, rendimiento y superficie presentan alzas respecto a 1997, de este modo la producción de avena se vio incrementada en 40.496 qmt, el rendimiento lo hizo en 11,1 qmt / ha y la superficie de cultivo, respecto a 1997, se expandió 67,5 ha. La ZM1, también tuvo un alza en los parámetros productivos entre el periodo intercensal, incrementó su producción en $16.718 \mathrm{qmt}$, el rendimiento en 5,7 qmt/ha y la superficie de cultivo en 362,1 ha. La ZB, en tanto, incrementó su producción en 31.126 qmt y el rendimiento por hectárea en 21,4qmt/ha, aún cuando la superficie de cultivo disminuyó 316,5 ha respecto a 1997.

En el caso de la cebada cervecera, la ZA experimentó un alza en producción, rendimiento y superficie de cultivo, en cambio el resto de las comunas presentaron una reducción sustantiva de producción y superficie de cultivo. De este modo, la ZM2 disminuyó su producción y superficie en 1997 de 53.402 qmt en 1.263,9 ha a 21.945 qmt en 451,6 ha, para el año 2007. La ZM1, bajó su producción de 4.960 qmt a 1.822 qmt en 2007 y la extensión de cultivos de 114 ha a 41 ha en 2007. La ZB, disminuyó su producción de 23.348 qmt a 10.944 qmt en 2007 y la superficie total de cultivo de 711,9 ha a 267,1 ha en 2007 . El rendimiento del cultivo para el periodo analizado aumentó para todas las zonas de la subcuenca del río Quepe.

La cebada forrajera en la ZA presentó una caída en producción y superficie del cultivo, disminuyendo la primera de $14.781 \mathrm{qmt}$ en 1997 a 11.832 qmt en 2007, mientras que la segunda lo 
hizo de 360,8 ha en 1997 a 246,0 ha a 2007. Misma situación siguió la ZM2, donde su producción descendió en 7.323 qmt respecto a 1997 y la superficie sembrada lo hizo en 214,8 ha. La ZM1, en cambio, mantuvo al alza la producción, el rendimiento y la superficie de cultivo, que para el año 2007 alcanzaron 2.572 qmt, 42,4 qmt/ha y 60,6 ha, respectivamente. La ZB presentó una leve alza en su producción, pasando de producir 1.733 qmt en 1997 a producir 1750 qmt en 2007, en paralelo la superficie de cultivo disminuyó 30,9 ha. Respecto del rendimiento, éste presentó una leve baja sólo en la ZM2, incrementándose en el resto de la cuenca, llegando incluso a duplicarse en la ZM1.

En la Araucanía la superficie cultivada con los principales cereales regionales; trigo, avena, cebada, maíz y triticale presentó un balance negativo, perdiendo $28.604,5$ ha de superficie respecto al VI censo agropecuario y forestal (ICET-ODEPA). A escala nacional, se repite la misma situación respecto a 1997, informándose caídas en la extensión territorial de cultivos esenciales (INE-ODEPA, 2007; INE, 2008; ODEPA, 2009). La superficie cubierta con cereales disminuyó en 168.707 ha, mientras que las plantas forrajeras anuales y perennes perdieron 10.900 ha y las leguminosas y tubérculos presentaron una reducción de 5.649 ha de superficie cultivada (ODEPA, 2009).

Debido a que la masa bovina constituye un $60 \%$ de la masa ganadera total del área de estudio, es relevante señalar que la masa bovina y el número de productores bovinos a nivel nacional y regional ha disminuido (INE, 2008), pudiendo ello asociarse a la competencia de los mercados extranjeros del rubro y sus estándares de calidad más elevados (INE-ODEPA, 2007). Las variaciones de masa ganadera obedecen también a la necesidad de exportación y consumo, como el caso del ganado porcino, el cual incrementó en $66,9 \%$ su masa a nivel nacional por esta razón (INE-ODEPA, 2007).

La región ha mantenido un auge constante en la actividad forestal, aumentando en un $20 \%$ la cobertura forestal exótica para el periodo analizado respecto de periodo anterior (19801993). Entre los años 2000 y 2007 la región exhibe el primer lugar nacional en cuanto a superficie forestada (CONAF(a); ODEPA, 2009). Estas estadísticas nacionales son coincidentes con lo señalado por Aguayo et al (2009) en cuanto a situar la década del ochenta como el comienzo de la expansión progresiva de especies exóticas de rápido crecimiento en todo el territorio nacional. A escala regional, prácticamente la mitad del suelo dedicado a fines productivos está utilizada con especies forestales: plantaciones (27\%) y bosque nativo (22\%) (INE-ODEPA, 2007). A escala nacional, CONAF (2011) señala que la cobertura forestal alcanza el $20,7 \%$ de la superficie nacional (15,5 millones de hectáreas), de la cual, el $83 \%$ corresponde a bosque nativo y $16 \%$ a plantaciones artificiales, incluyéndose a Chile como país con práctica forestal intensiva (Hansen et al., 2013).

INE-ODEPA (2007) señala que, como resultado de un fuerte incremento en la superficie de otros usos de suelo (forestal, industrial, praderas naturales), los suelos de cultivo han mantenido una sostenida baja en su superficie nacional, pasando de 3.3 millones de hectáreas en 1976 a alrededor de 2 millones de hectáreas en 2007. Sumado a esto, ODEPA (2009) realza la fuerte expansión de la agricultura de exportación y de cultivos intensivos e industriales (INE, 2007), en desmedro de la superficie de cultivos tradicionales como cereales, forrajeras, leguminosas y tubérculos. Este crecimiento de la agricultura de exportación a expensas de la agricultura tradi- 
cional, puede asociarse al crecimiento del mercado de alimentos procesados, a cambios en los patrones de consumo de la población, a ventajas competitivas relacionadas con la calidad por sobre la cantidad y a la mayor rentabilidad en comparación a los rubros tradicionales, e.g. el rubro frutícola, el cual está aumentando el interés de los productores de la Araucanía y cada vez atrae más inversionistas a la actividad (ODEPA-CIREN, 2012).

\section{Análisis de cambio de cobertura de suelo periodo 1994-2007}

La matriz de cambio muestra que el $14,2 \%$ de la superficie de la subcuenca, equivalente a $22.773,6$ ha, experimentó cambios en todas las coberturas de suelo construidas para el área de estudio. El cambio se manifiesta en pequeñas áreas en toda la subcuenca, concentrándose principalmente en el centro y sur de la ZA (Figura $N^{\circ} 3$ ). Las coberturas que manifestaron los mayores cambios respecto al año 1994 correspondieron a "plantaciones forestales", "cultivos y praderas naturales" y "bosque nativo y matorrales" (Cuadro $N^{\circ} 1$ ).

\section{Figura $\mathrm{N}^{\circ} 3$}

Superficie de cambio de cobertura de suelo periodo 1997-2007 en la subcuenca precordillerana del río Quepe, región de La Araucanía, Chile.

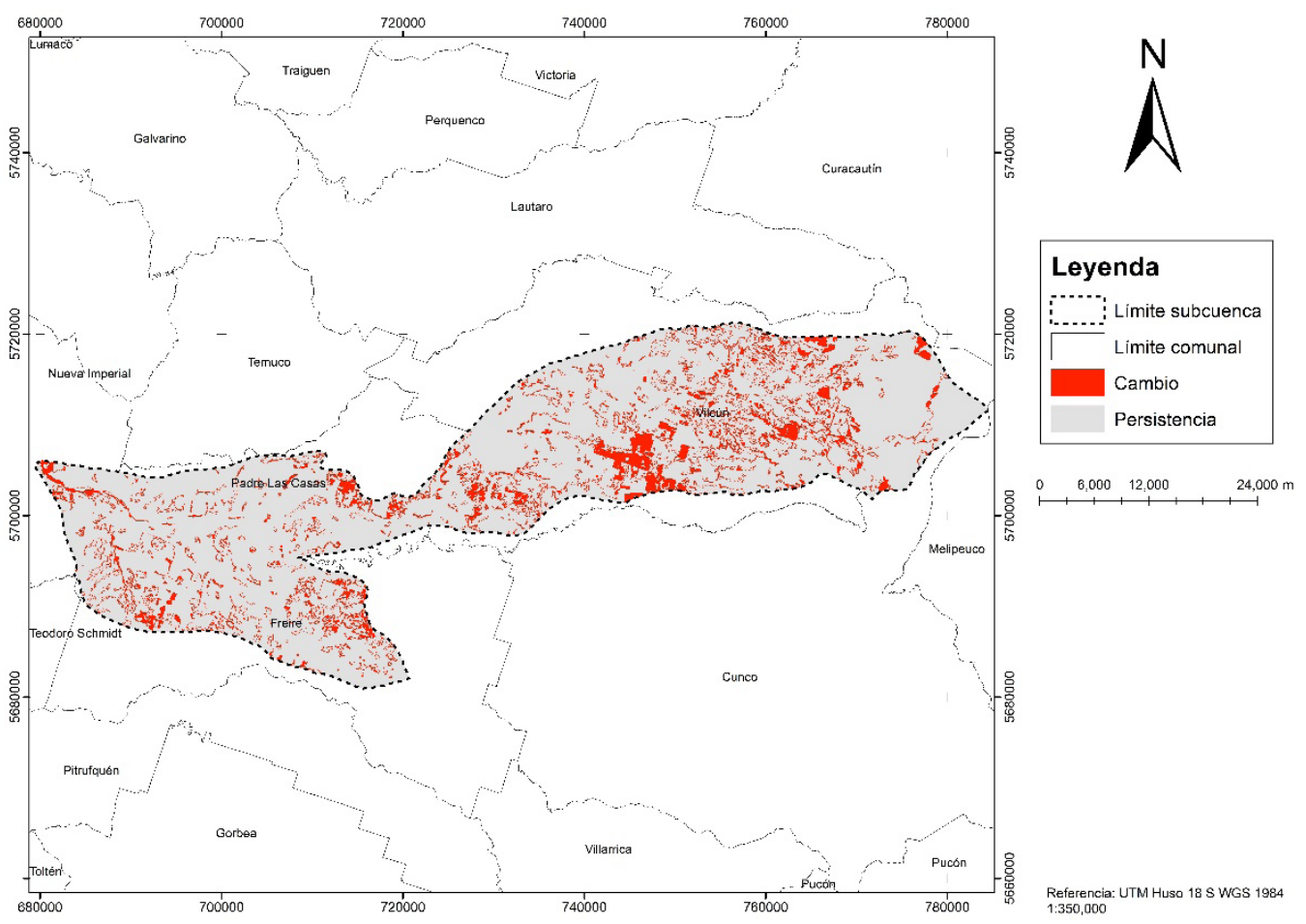

Fuente: Elaboración propia. 
Cuadro $\mathrm{N}^{\circ} 1$

Matriz de cambio uso de suelo subcuenca del río Quepe, región de La Araucanía. Cada columna corresponde a las hectáreas totales de cobertura en 1994, cada fila corresponde a las hectáreas totales de cobertura en 2007

\begin{tabular}{|c|c|c|c|c|c|c|c|c|}
\hline & & \multicolumn{7}{|c|}{ Año 1994} \\
\hline & & $\begin{array}{c}\text { Bosque } \\
\text { nativo y } \\
\text { matorrales }\end{array}$ & $\begin{array}{c}\text { Cultivos y } \\
\text { praderas } \\
\text { naturales }\end{array}$ & $\begin{array}{c}\text { Plantaciones } \\
\text { forestales }\end{array}$ & $\begin{array}{l}\text { Praderas } \\
\text { perennes }\end{array}$ & $\begin{array}{c}\text { Otras } \\
\text { coberturas }\end{array}$ & Total 2007 & $\begin{array}{c}\text { Ganancia } \\
\text { bruta }\end{array}$ \\
\hline \multirow{7}{*}{ 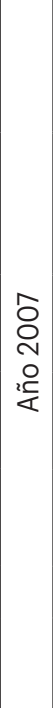 } & $\begin{array}{l}\text { Bosque } \\
\text { nativo y } \\
\text { matorrales }\end{array}$ & $31.923,2$ & $6.410,9$ & 778,3 & $1.544,9$ & 330,6 & 40.988 & $9.064,8$ \\
\hline & $\begin{array}{l}\text { Cultivos y } \\
\text { praderas } \\
\text { naturales }\end{array}$ & $3.206,6$ & 90.126 & 307,2 & 450,5 & 191,9 & $94.282,4$ & $4.156,4$ \\
\hline & $\begin{array}{l}\text { Plantaciones } \\
\text { forestales }\end{array}$ & $1.673,2$ & $4.986,1$ & $4.389,8$ & 331,7 & 23,5 & $11.404,6$ & $7.014,7$ \\
\hline & $\begin{array}{l}\text { Praderas } \\
\text { perennes }\end{array}$ & $1.207,1$ & 237,3 & 288,3 & $7.394,7$ & 53 & $9.180,7$ & $1.785,9$ \\
\hline & $\begin{array}{l}\text { Otras } \\
\text { coberturas }\end{array}$ & 189,9 & 512,1 & 21,5 & 28 & $3.753,3$ & 4.505 & 751,7 \\
\hline & Total 1994 & $38.200,1$ & $102.272,5$ & $5.785,4$ & 9.750 & $4.352,5$ & $160.360,8$ & \\
\hline & $\begin{array}{l}\text { Pérdida } \\
\text { bruta }\end{array}$ & $6.276,9$ & $12.146,5$ & $1.395,5$ & $2.355,30$ & 599,2 & & \\
\hline
\end{tabular}

Fuente: Elaboración propia.

Según la matriz de cambio, la cobertura "bosque nativo y matorrales" experimentó una ganancia bruta de $9.064,8$ ha y una pérdida bruta de $6.276,9$ ha, cuya diferencia representa un incremento de $2.787,8$ ha $(7,3 \%)$ entre el periodo $1994-2007$. Para "cultivos y praderas naturales", la ganancia bruta de esta cobertura fueron $4.156,4$ ha, mientras que la pérdida bruta fue de 12.146,5 ha. Para el periodo de análisis, esta cobertura registró la pérdida más alta de superficie, superando casi tres veces a la ganancia bruta. En efecto, el cambio neto tuvo una reducción de -7.990,1 ha $(-7,8 \%)$ al año 2007 en comparación con el año 1994.

La cobertura "plantaciones forestales" es la que presenta la mayor ganancia bruta después de "bosque nativo y matorrales" y con el mayor cambio neto de todas las coberturas de la subcuenca. Los valores de ganancia y pérdida brutas de superficie son $7.014,7$ ha y $1.395,5$ ha, respectivamente. En tanto, el cambio neto asciende a $5.619,1$ ha, un $97,1 \%$ de superficie superior a la ocupada en 1994, sumando en 2007 la magnitud de 11.404,6 ha de plantaciones forestales en la subcuenca.

Las "praderas perennes" de la subcuenca ganaron $1.785,9$ ha de superficie y perdieron $2.355,3$ ha, lo que significa un cambio neto de $-5,8 \%$, que equivale a una reducción de $-569,3$ ha (Cuadro $\left.N^{\circ} 1\right)$. En tanto, "otras coberturas" constituye la categoría con menor modificación y superficie de la subcuenca. Su ganancia bruta de superficie fue de 751,7 ha y su pérdida bruta alcanzó 599,2 
ha. Paralelamente, el cambio neto de la cobertura fue 3,5\%, que significa una ganancia de 152 ha respecto al año 1994.

El análisis de cambio de cobertura para el periodo 1994-2007 en la subcuenca del río Quepe muestra que "cultivos y praderas naturales" presentó una disminución de 7,8\% en su superficie. Estos resultados son concordantes con la situación regional, en la cual, según la última actualización de cambios del catastro de recursos vegetacionales nativos de Chile, la Araucanía disminuyó en $12,5 \%$ su superficie de terrenos agrícolas (CONAF-CONAMA, 2009). En paralelo, ICET-ODEPA indica que la disminución de la superficie de cultivos anuales y permanentes fue de $11,3 \%$ entre el periodo 1997-2007, situación que se asemeja a la agrupación de comunas que componen la subcuenca del rio Quepe, donde el área ocupada por cereales disminuyó 20\% entre 1997 y 2007 (INE, 2008; ICET-ODEPA). Paralelamente, la superficie de plantaciones forestales de la subcuenca aumentó 97,1\% entre el período 1994-2007. A escala regional, el VII censo agropecuario y la actualización del catastro para la Araucanía señalan un aumento de $32 \%$ y 62,9\%, respectivamente para esta cobertura (CONAF-CONAMA, 2009).

La disminución de la cobertura agropecuaria en la subcuenca se debe fundamentalmente al avance de la cobertura de plantaciones forestales sobre la primera y al consiguiente cambio de uso productivo. En la región, esta situación se reafirma para el periodo 2007-2013 en el cuál continúa incrementándose la superficie bajo uso "plantaciones forestales" desde usos "terrenos agrícolas", "praderas y matorrales" y "bosque nativo" (UACH-UFRO-CONAF, 2014). Para el periodo analizado en la subcuenca, la cobertura "cultivos y praderas naturales" cede la mayor proporción de superficie a la cobertura "plantaciones forestales". Este incremento en la cobertura forestal se explica principalmente por: la fuerte inversión regional capitalizada en la industria de la madera y el papel (75\%) a partir del año 2000, estando por sobre lo consignado para la industria agrícola $(10,7 \%)$ y silvícola $(10,1 \%)$ (CONAF-CONAMA, 2009) y por el incentivo estatal a la actividad mediante la Ley de Bosques y el decreto Ley 701 (Aguayo et al., 2009; Carmona \& Nahuelhual, 2012). En este sentido, al analizar las estadísticas nacionales forestales, se observa que entre los años 2002 y 2006 la superficie bonificada forestada representa más de $50 \%$ del total regional forestado y reforestado, expandiéndose notoriamente la superficie cubierta con Eucalyptus Globulus, pasando de 12.408 hectáreas en el año 2001 a 21.726 hectáreas en el año 2006 (CONAF(a)). Similar situación ha sido reportada para ecosistemas precordilleranos de la región del Maule, donde la cobertura "plantaciones forestales" aumenta en un 500\% en detrimento de "bosque nativo" y "terrenos agrícolas" en un periodo de 14 años (Altamirano \& Lara, 2010). A nivel nacional, CONAF(b) consigna el aumento en la superficie con plantación forestal con especies exóticas de 300.000 hectáreas en 1970 a 3.047 millones de hectáreas en 2016. Como señala Barbier et al. (2010), procesos de aforestación ocurren cuando los propietarios de la tierra y la industria forestal incrementan la inversión en más plantaciones y manejo de bosques secundarios para fines comerciales.

La superficie total del cambio neto en la subcuenca es dominada en un 79,5\% por las coberturas "cultivos y praderas naturales" y "plantaciones forestales". Estos resultados coinciden con lo reportado por varios estudios en cuanto a señalar que la actividad agrícola y forestal representan los principales factores que han modificado la cobertura de suelo en el centro-sur de Chile (Donoso \& Lara, 1996; Azócar \& Sanhueza, 1999; Cisterna et al., 1999; Aguayo et al., 2009; CONAF, 2011). Junto con ello, los datos obtenidos muestran una gran presión de cambio de uso de suelo concentrada en una fracción acotada de la subcuenca (14\%) en la que domina el cambio hacia plantación 
de especies forestales exóticas, impactando la sostenibilidad en el largo plazo, la conservación del suelo, el secuestro de carbono y modificación del clima local (Baldi \& Paruelo, 2008).

Esta situación de aumento de la superficie de uso forestal a expensas de la superficie de uso agrícola y pecuario ocurre también en países asiáticos (China, India and Vietnam) (Barbier et al., 2010). En esos casos, al igual que en Chile, se evidencia la acelerada recuperación en el uso forestal (incluido los monocultivos forestales) mediante políticas forestales de incentivo para la recuperación de suelos degradados. Sin duda que el modelo forestal chileno ha permitido una exitosa expansión de la plantación forestal de especies exóticas que no ha sido equiparada con la conservación y restauración del bosque nativo (Miranda et al., 2017).

La cobertura "bosque nativo y matorral" presenta una ganancia de 2.787,9 ha en el periodo analizado, a una tasa de 214 ha año-1. En este caso, dada la clasificación de la cobertura, no es posible distinguir cuanto corresponde realmente a ganancia efectiva en reforestación con especies forestales nativas. Según la información regional y local levantada en este estudio, es posible inferir que, para esta cobertura, la ganancia se explica por el dominio de la composición matorral producto de la pérdida de bosque nativo por deforestación impulsada por la actividad forestal y por el abandono de suelos de uso agrícola y pecuario los que comienzan a ser recolonizados iniciándose la sucesión a matorral. Este abandono de los suelos de uso agrícola, postulamos, se debe principalmente a la migración campo ciudad y posiblemente fuera de la región. En esta línea, en Chile, Miranda et al. (2017) reportan que entre las regiones de Valparaíso y Los Lagos $\left(33^{\circ} \mathrm{S}\right.$ a $42^{\circ} \mathrm{S}$ ), zona considerada un hotspot en biodiversidad, ha existido una pérdida continua de bosques en los últimos 40 años (1970-2010), donde la conversión de bosque nativo a matorral representa la tendencia principal de cambio de cobertura.

Por su parte, CONAF (2014) señala que, para el periodo 1994-2011 en la región de la Araucanía, junto con el sostenido crecimiento en el uso de suelo para plantación forestal exótica se ha mantenido el número de pobres; además, en el periodo 2002-2012 se reporta un descenso en la población total regional, en el cual la población rural disminuyó sistemáticamente, siendo al 2012 la mitad registrada en el censo de población de 1992. Para Donoso \& Otero (2005), una de las mayores contradicciones del sector forestal chileno es que, a pesar del aumento en las exportaciones forestales, principalmente en las regiones del Maule, Bío-Bío y la Araucanía, la población de esas regiones no ha visto mejorada su calidad de vida. Esta situación regional se contrapone con lo señalado por IIED (2004) el que destaca el rol de la plantación forestal en la reducción de la pobreza en países en desarrollo.

La distribución de la cobertura de suelo en un territorio es más compleja que la sola reducción de la superficie de una cobertura original, implica también impactos en los flujos de nutrientes, agua y energía en el ecosistema (Baldi \& Paruelo, 2008; Grau \& Aide, 2008). Por su parte, Bremer \& Farley (2010), en su revisión de varios grupos de datos cuantitativos ya publicados, señalan que la plantación forestal contribuye positivamente a la biodiversidad cuando es establecida en suelos degradados y no en bosque nativo, praderas o matorrales y cuando se prefiere el uso de especies arbóreas nativas en vez de especies exóticas. Bae et al. (2012) realzan la necesidad de desarrollar indicadores que reflejen cambios en la biodiversidad y otros componentes del ecosistema diferenciados para bosques primarios y secundarios. 
Consiguientemente, en la subcuenca precordillerana del río Quepe, la evolución temporal en el uso de suelo implica que la cubierta vegetal ha sido drásticamente alterada dado el aumento en la presión antrópica sobre el sistema, reflejado ello en una dinámica productiva intensa en el territorio (agrícola, pecuaria y forestal). Este cambio en la cobertura responde a una modificación del valor del precio del suelo de uso agrícola respecto al suelo de uso forestal, impulsado por factores exógenos (mercados, políticas nacionales, incentivos económicos, etc.), disminuyendo su valor el primero en la medida que el retorno económico de la inversión con fines agropecuarios es menor (Babier et al., 2010). En este sentido, el comportamiento dinámico del cambio de cobertura y uso de suelo en la subcuenca se corresponde con lo señalado por Lambin \& Meyfroidt (2010) en cuanto a que el cambio de cobertura de suelo no tiene un comportamiento lineal, sino que se asocia tanto a cambios biofísicos como socioeconómicos en una serie sucesiva de transiciones temporales. Dado ello, en la subcuenca analizada, la distribución y cambio temporal de la cobertura de suelo responde principalmente a factores socioeconómicos y no se relaciona directamente con la calidad intrínseca de los suelos, es decir, suelos de uso preferentemente agrícola están siendo transformados a uso forestal. En este contexto, es prioritario generar un programa de evaluación del estado y calidad del suelo tal que permita inferir su sustentabilidad futura.

\section{Validación}

El índice de kappa presentó un valor de 0,84 para las categorías construidas en este trabajo, lo que sugiere una precisión del $84 \%$ mejor del mapa-realidad observada, mientras que la matriz de confusión proporcionó un valor de precisión global de la clasificación de 87\%. Landis \& Kock (1977), sugieren un valor de Kappa entre 0,61 y 0,80 y un valor para la precisión de la clasificación global de $85 \%$ como mínimo estandarizado y aceptado (Foody, 2002). Por tanto, se afirma que la cartografía producida en este trabajo no es producto del azar y las categorías de cobertura se pueden discriminar en el área de estudio (Arenas et al., 2010).

\section{Conclusiones}

Los suelos de uso agrícola muestran una reducción en su superficie nacional, regional y local. En la subcuenca precordillerana del río Quepe, dicha disminución se atribuye principalmente a la expansión de la actividad forestal, la que duplicó su superficie entre 1994 y 2007 en desmedro de los suelos para uso de cultivos y praderas.

Para el periodo analizado, 1994-2007, las coberturas que presentaron aumento de superficie fueron la categoría "plantaciones forestales" con una tasa positiva de 432 ha año-1, seguido de "bosque nativo y matorral" con una ganancia de 214 ha año-1. Las coberturas que presentaron pérdida de superficie fueron la categoría "cultivos y praderas naturales" con una tasa de reducción de 614 ha año-1 y la categoría "praderas perenne" con una pérdida de 44 ha año-1. En la subcuenca, el cambio de cobertura se concentra en el $14 \%$ de la superficie total de la misma, representado el uso intensivo del suelo en esas áreas.

En la subcuenca, la distribución de la cobertura de suelo y su cambio en el periodo 1994-2007 responde principalmente a factores socioeconómicos externos (aumento de la rentabilidad de la actividad forestal, migración campo ciudad y abandono de suelos de uso agropecuario) y no 
se relaciona directamente con la calidad intrínseca de los suelos bajo uso productivo, es decir, suelos de uso agrícola están siendo transformados a uso forestal.

Dado los resultados obtenidos, fue posible identificar territorialmente zonas de cambio de cobertura de suelo en el área de estudio y determinar la dinámica de uso para esas unidades, reafirmándose la necesidad de analizar el cambio de cobertura del suelo en un territorio, previo a la identificación de las potencialidades del recurso para soportar nuevas actividades productivas.

\section{Referencias bibliográficas}

AGUAYO, M.; PAUCHARD, A.; AZÓCAR, G. \& PARRA, O. Cambio del uso del suelo en el centro sur de Chile a fines del siglo XX: Entendiendo la dinámica espacial y temporal del paisaje. Revista Chilena de Historia Natural, 2009, Vol. 82, p. 361-374.

ALTAMIRANO, A. \& LARA, A. Deforestación en ecosistemas templados de la precordillera andina del centro-sur de Chile. Bosque, 2010, Vol. 31, N¹, p. 53-64.

ARENAS, S.; HAEGER, J. \& JORDANO, D. Aplicación de técnicas de teledetección y gis sobre imágenes quickbird para identificar y mapear individuos de peral silvestre (Pyrus bourgeana) en bosque esclerófilo mediterráneo. Revista de Teledetección, 2010, Vol. 35, p. 55-71.

AZÓCAR, G. \& SANHUEZA, R. Evolución del uso del suelo en las cuencas hidrográficas de las lagunas de la comuna de San Pedro de la Paz, región del Biobío: análisis histórico y tendencias. Revista Geográfica de Chile Terra Australis, 1999, Vol. 44, p. 63-78.

BAE, J.; WON JOO, R. \& KIM, Y. Forest transition in South Korea: Reality, path and drivers. Land Use Policy, 2012, Vol. 29, p. 198-207.

BALDI, G. \& PARUELO, J. Land-use and land cover dynamics in South American temperate grasslands. Ecology and Society, 2008, Vol. 13, N², p. 6.

BARBIER, E.; BURGESS, J. \& GRAINGER, A. The forest transition: Towards a more comprehensive theoretical framework. Land Use Policy, 2010, Vol. 27, p. 98-107.

BRAIMOH, A.K. Random and systematic land-cover transitions in northern Ghana. Agriculture, Ecosystems \& Environment, 2006, Vol. 113, №1-4, p. 254-263.

BREMER, L. \& FARLEY, K. Does plantation forestry restore biodiversity or create green deserts? A synthesis of the effects of land-use transitions on plant species richness. Biodiversity Conservation, 2010, Vol. 19, p. 3893-3915.

CADE-IDEPE. Diagnóstico y clasificación de los cursos y cuerpos de agua según objetivos de calidad, cuenca del río Imperial. Temuco: Dirección General de Aguas, 2004, 137 p.

CARMONA, A. \& NAHUELHUAL, L. Combining land transitions and trajectories in assessing forest cover change. Applied Geography, 2012, Vol. 32, p. 904-915. 
CHAPIN, M.; LAMB, Z. \& THRELKELD, B. Mapping Indigenous Lands. Annual Review of Anthropology, 2005, Vol. 34, p. 619-38.

CHUVIECO, E. Teledetección ambiental: La observación de la Tierra desde el espacio. Barcelona: Editorial Ariel S.A, 2008.

CISTERNA, M.; MARTÍNEZ, P.; OYARZÚN, C. y DEBELS, P. Caracterización del proceso de reemplazo de vegetación nativa por plantaciones forestales en una cuenca lacustre de la cordillera de Nahuelbuta, VIII Región, Chile. Revista Chilena de Historia Natural, 1999, Vol. 72, p. 541-556.

CORPORACION NACIONAL FORESTAL (CONAF). Catastro de los recursos vegetacionales nativos de Chile: monitoreo de cambios y actualizaciones período 1997-2011. Santiago de Chile: Ministerio de Agricultura, 2011, $28 \mathrm{p}$.

CORPORACION NACIONAL FORESTAL (CONAF). Plantaciones y pobreza en comunas forestales: Forestación y estilo de desarrollo. 2014. Disponible en Internet: http://www.conaf.cl/centro-de-documentacion/nuestros-bosques/

CORPORACION NACIONAL FORESTAL (CONAF). Superficies catastros usos de suelos y recursos vegetacionales. S/f. (a). Disponible en Internet: http://sit.conaf.cl/

CORPORACION NACIONAL FORESTAL (CONAF). Estadísticas forestales. S/f. (b) Disponible en Internet: http://www.conaf.cl/nuestros-bosques/bosques-en-chile/estadisticas-forestales/

CORPORACION NACIONAL FORESTAL (CONAF) - COMISIÓN NACIONAL DEL MEDIO AMBIENTE (CONAMA). Catastro de uso del suelo y vegetación, monitoreo y actualización para la Región de La Araucanía. Santiago de Chile: Ministerio de Agricultura, 2009.

CORPORACION NACIONAL FORESTAL-COMISIÓN NACIONAL DEL MEDIO AMBIENTE-BANCO INTERNACIONAL DE RECONSTRUCCIÓN Y FOMENTO (CONAF-CONAMA-BIRF). Catastro y evaluación de los recursos vegetacionales nativos de Chile: Informe nacional con variables ambientales. Santiago de Chile: Corporación Nacional Forestal, 1999.

DIRECCIÓN GENERAL DE AGUAS (DGA). Análisis de disponibilidad recursos hídricos superficiales cuenca río Quepe. Temuco: Departamento de Administración de Recursos Hídricos, Ministerio de Obras Públicas, 2004, 90 p.

DI CASTRI, F. \& HAJEK, E. Bioclimatología de Chile. Santiago de Chile: Ediciones Universidad Católica de Chile, 1976.

DONOSO, C. \& LARA, A. Utilización de los bosques nativos en Chile: pasado, presente y futuro. En: ARMESTO, JJ., C. VILLAGRÁN, y M.K. ARROYO (editores). Ecología de los bosques nativos de Chile. Santiago, Chile: Editorial Universitaria, 1996, p. 363-387.

DONOSO, P. \& OTERO, L. Hacia una definición de país forestal: ¿Dónde se sitúa Chile?. Bosque, 2005, Vol. 26, N³, p. 5-18. 
FARLEY, K. Grasslands to Tree Plantations: Forest Transition in the Andes of Ecuador. Annals of the Association of American Geographers, 2007, Vol. 97, № 4, p. 755-771.

FOODY, G. Status of land cover classification accuracy assessment. Remote Sensing of Environment, 2002, Vol. 80, p. 185-201.

FOLEY, J.; DEFRIES, R.; ASNER, G.; BARFORD, G. \& BONAN G. Global consequences of land use. Science, 2005, Vol. 309, p. 570-574.

GRAU, H. \& AIDE, Y. Globalization and land-use transitions in Latin America. Ecology and Society, 2008, Vol. 13, N², p. 16.

HANSEN, M.; POTAPOV, P.; MOORE, R.; HANCHER, M.; TURUBANOVA, S.; TYUKAVINA, A.; THAU, D.; STEHMAN, S.; GOETZ, S.; LOVELAND, T.; KOMMAREDDY, A.; EGOROV, A.; CHINI, L.; JUSTICE, C. \& TOWNSHEND, J. High-Resolution Global Maps of 21st-Century Forest Cover Change. Science, 2013, Vol. 342, Nº160, p. 850-853.

HOOKE, R.; MARTÍN-DUQUE, J. \& PEDRAZA, J. Land transformation by humans: A review. GSA Today, 2012, Vol. 22, Nº12, p. 4-10.

ICET-ODEPA. Sistema de consulta estadístico territorial: censo agropecuario. S/f. Disponible en Internet: http://icet.odepa.cl/

IIED. Plantation, Privatization, Poverty and Power: Changing ownership and management of state forests. Londres, UK: Earthscan Pub., 2004, 328 p.

ILUSTRE MUNICIPALIDAD DE NUEVA IMPERIAL. Plan de desarrollo comunal 2011-2014 de la comuna de Nueva Imperial. Nueva Imperial: Municipio de Nueva Imperial, 2010.

ILUSTRE MUNICIPALIDAD DE VILCÚN. Plan de desarrollo comunal 2011-2014 Vilcún, actualización. Vilcún: Municipio de Vilcún, 2010.

INSTITUTO NACIONAL DE ESTADÍSTICAS (INE). Enfoque estadístico: VII censo agropecuario y forestal. Santiago de Chile: Unidad de Ediciones Instituto Nacional de Estadísticas, 2008.

INSTITUTO NACIONAL DE ESTADÍSTICAS (INE). Censo Agropecuario y Forestal 2007, resultados por comuna. 2017. Disponible en Internet:

http://www.ine.cl/canales/chile_estadistico/censos_agropecuarios/censo_agropecuario_07_comunas.php/

INSTITUTO NACIONAL DE ESTADÍSTICAS- OFICINA DE ESTUDIOS Y POLÍTICAS AGRARIAS (INE-ODEPA). Enfoque estadístico: VII censo nacional agropecuario y forestal. Santiago de Chile: Ministerio de Agricultura, 2007.

LAMBIN, E. \& MEYFROIDT, P. Land use transitions: Socio-ecological feedback versus socio-economic change. Land Use Policy, 2010, Vol. 27, p. 108-118. 
LANDIS, J. \& KOCK, G. The measurement of observer agreement for categorical data. Biometrics, 1977, Vol. 33, p. 159-174.

MAASS, J. y COTLER, H. Protocolo para el manejo de ecosistemas en cuencas hidrográficas. En: COTLER H. (compilador). El manejo integral de cuencas en México: estudios y reflexiones para orientar la política ambiental. México: Secretaría del Medio Ambiente y Recursos Naturales, Instituto Nacional de Ecología, Segunda Edición, 2008, p. 41-64.

MARÍN, S.; NAHUELHUAL, L.; ECHEVERRÍA, C. \& GRANT, W. Projecting landscape changes in southern Chile: simulation of human and natural processes driving land transformation. Ecological Modelling, 2011, Vol. 222, p. 2841-2855.

MATHER, A. Recent Asian forest transition in relation to forest transition theory. International Forestry Review, 2007, Vol. 9, № 1, p. 491-502.

MIRANDA, A.; ALTAMIRANO, A.; CAYUELA, L.; LARA, A. \& GONZÁLEZ, M. Native forest loss in the Chilean biodiversity hotspot: revealing the evidence. Regional Environmental Change, 2017, Vol. $17, N^{\circ} 1$, p. 285-297.

MOHAMMAD, A. \& MOHAMMAD, M. The impact of vegetative cover type on runnof and soil erosion under different land uses. Catena, 2010, Vol. 81, p. 97-103.

NÁJERA, O.; BOJÓRQUEZ, J.; CIFUENTES, J. y MARCELEÑO, S. Cambio de cobertura y uso del suelo en la cuenca del río Mololoa, Nayarit. Biociencias, 2010, Vol. 1, Nº1, p. 19-29.

OFICINA DE ESTUDIOS Y POLÍTICAS AGRARIAS (ODEPA). Análisis del VII censo nacional agropecuario y forestal. Santiago de Chile: Ministerio de Agricultura, 2009, $10 \mathrm{p}$.

OFICINA DE ESTUDIOS Y POLÍTICAS AGRARIAS- CENTRO DE INFORMACIÓN DE RECURSOS NATURALES (ODEPA-CIREN). Catastro frutícola: región de La Araucanía. Santiago de Chile: Ministerio de Agricultura, 2012.

OEHL, F.; LACZKO, E.; BOGENRIEDER, A.; STAHR, K.; BÖSCH, R.; VAN DER HEIJDEN, M. \& SIEVERDING, E. Soil type and land use intensity determine the composition of arbuscular mycorrhizal fungal communities. Soil Biology and Biochemistry, 2010, Vol. 42, N5, p. 724-738.

OUYANG, W.; SKIDMORE, A.; HAO, F. \& WANG, T. Soil erosion dynamics response to landscape patterns. Science of the Total Environment, 2010, Vol. 408, p. 1358-1366.

PONTIUS, R.; SHUSAS, E. \& McEACHERN, M. Detecting important categorical land changes while accounting for persistence. Agriculture, Ecosystems \& Environment, 2004, Vol. 101, p. 251-268.

RAMANKUTTY, N. \& FOLEY, J. Estimating historical changes in global land cover: Croplands from 1700 to 1992. Global Biogeochemical Cycles, 1999, Vol. 13, p. 997-1027.

REYES, H.; AGUILAR, R.; AGUIRRE, R. y TREJO, V. Cambios en la cubierta vegetal y uso del suelo en el área del proyecto Pujal Coy, San Luis Potosí, México, 1973-2000. Investigaciones Geográficas, 2006, Vol. 59, p. 26-42. 
RUDEL, T.; COOMES, O.; MORAN, E.; ACHARD, F.; ANGELSEN, A.; XU, J. \& LAMBIN, E. Forest transitions: Towards a global understanding of land use change. Global Environmental Change, 2005, Vol. 15, p. 23-31.

UNIVERSIDAD AUSTRAL DE CHILE-UNIVERSIDAD DE LA FRONTERA-CORPORACIÓN NACIONAL FORESTAL (UACH-UFRO-CONAF). Monitoreo de Cambios, Corrección Cartográfica y Actualización Del Catastro de los Recursos Vegetacionales Nativos de la región de La Araucanía. Temuco: CONAF, 2014. 
